DDS - Die Deutsche Schule 111. Jahrgang 2019, Heft 4, S. 471-478 https://doi.org/10.31244/dds.2019.04.09

(c) 2019 Waxmann

Colin Cramer

\title{
Multiparadigmatische und meta-reflexive Lehrerbildung
}

Begründungen, Gemeinsamkeiten und Differenzen, Perspektiven

\section{Zusammenfassung}

Dieser Beitrag reagiert neben den Diskussionsbeiträgen von Jörg Schlömerkemper und Nils Berkemeyer in dieser Ausgabe auf den Diskussionsbeitrag: Heinrich, M., Wolfswinkler, G., van Ackeren, I., Bremm, N., \& Streblow, L. (2019). Multiparadigmatische Lehrerbildung. Produktive Auswege aus dem Paradigmenstreit? DDS - Die Deutsche Schule, 111 (2), 244-259. https://doi.org/10.31244/dds.2019.02.10

Schlüsselwörter: Lehrerbildung, Professionalisierung, Paradigmen, Professionalität, Professionstheorie

\section{Multiparadigmatic and Meta-reflexive Teacher Education}

Rationales, Commonalities and Differences, Perspectives

\begin{abstract}
This article, besides the articles by Jörg Schlömerkemper and Nils Berkemeyer in this issue, reacts to the contribution: Heinrich, M., Wolfswinkler, G., van Ackeren, I., Bremm, N., \& Streblow, L. (2019). Multiparadigmatic Teacher Training. Productive Ways out of the Paradigm Dispute? DDS - Die Deutsche Schule, 111 (2), 244-259. https://doi.org/10.31244/dds.2019.02.10
\end{abstract}

Keywords: teacher training, professionalization, paradigms, professionalism, profession theory

\section{Ausgangslage}

Beinahe zeitgleich wurden zwei Texte publiziert, die Mehrperspektivität in der Lehrerbildung auf je spezifische Weise ausdifferenzieren. In Heft 2/2019 der Zeitschrift DDS - Die Deutsche Schule stellen Heinrich, Wolfswinkler, van Ackeren, Bremm \& Streblow erste Überlegungen zur Multiparadigmatischen Lehrerbildung an. Cramer, Harant, Merk, Drahmann \& Emmerich haben in Heft 3/2019 der Zeit- 
schrift für Pädagogik den professionstheoretischen Ansatz der Meta-Reflexivität auch in Bezug auf die Lehrerbildung entfaltet. Dieser Diskussionsbeitrag skizziert Begründungen, Gemeinsamkeiten und Differenzen sowie Perspektiven der beiden Ansätze.

Unter anderem folgende Entwicklungen sind für das Aufkommen der Erwägungen zu einer Mehrperspektivität in der Lehrerbildung relevant:

(1) Mit der Einführung von Standards für die Lehrerbildung (KMK, 2004) und verstärkt durch die „Qualitätsoffensive Lehrerbildung“ wird über Ziele von Lehrerbildung diskutiert. Dieser Verständigungsprozess evoziert „systematische Kämpfe um Deutungshoheit“ (Heinrich et al., 2019, S. 244). Doch die Erziehungswissenschaft kann schon aufgrund der Publikationslage kaum beanspruchen, „Leitdisziplin“ der Lehrerbildung zu sein (Cramer, 2018): Das Verhältnis von Lehrerausbildung (als berufsqualifizierendem Programm) und Erziehungswissenschaft (als forschender Disziplin) wird kontrovers diskutiert (Böhme, Cramer \& Bressler, 2018).

(2) In der „Qualitätsoffensive“ wurden historisch einmalig viele Personalstellen geschaffen, die „gute Ideen“ zur Entwicklung der Lehrerbildung einbringen. Daraus resultierende Maßnahmen verlaufen teils parallel oder sogar quer zu disziplinären bildungswissenschaftlichen und fachdidaktischen Diskursen. Um die als „Flickenteppich“ (Terhart, 2000, S. 27) charakterisierte Lehrerbildung nicht zu einer noch stärkeren Verinselung separierter Einzelinitiativen werden zu lassen, kommt die Frage nach Kohärenz in den Blick, also nach dem Zusammenhalt der Elemente des Lehrerbildungssystems (z.B. Abstimmung von Curricula zwischen Fächern oder Phasen; Glowinski, Borowski, Gillen, Schanze \& von Meien, 2018).

(3) Mehr als auf solch eine formell-institutionelle Kohärenz zielen die diskutierten Ansätze aber auf die informell-individuelle Kohärenz, also die Kohärenzstiftung durch die Akteure der Lehrerbildung selbst: Wie stellen Dozierende oder Fortbildende Bezüge zwischen Elementen her? Wie vermitteln Studierende gedanklich zwischen (widersprüchlichen) Elementen?

Sowohl das Programm der multiparadigmatischen Lehrerbildung als auch der Ansatz der meta-reflexiven Lehrerbildung sensibilisieren für die hier angerissenen Fragen. Beide Konzepte werden zunächst knapp skizziert, und im Anschluss werden Gemeinsamkeiten und Differenzen sowie Perspektiven für die weitere Diskussion aufgezeigt. 


\section{Multiparadigmatische Lehrerbildung}

Heinrich et al. (2019) folgen dem Anspruch zu zeigen, wie sich „unnötige Implementationshürden monoparadigmatischer Vereinseitigungen" (S. 243) durch multiparadigmatische Lehrerbildung mildern lassen. Eine gegenstandsorientierte und systematische Meta-Theoriebildung stehe noch aus; es werde zunächst nur eine Heuristik vorgestellt. Eine solche regulative Idee sei erforderlich, weil Diskussionen um Deutungshoheit unproduktiv seien und monoparadigmatische Verengungen mit Risiken einhergingen. Multiparadigmatische Lehrerbildung sei „alternativlos“ (ebd.).

Als heuristischen Ausgangspunkt für die theoretische Fundierung des Programmes wählt das Autor*innenteam die Grundidee des didaktischen Dreieckes (Lernende, Lehrende, Gegenstand). Seitens der Lernenden (Studierenden) wird auf Heterogenität und damit auf unterschiedliche Wege der Welterschließung rekurriert. Studierende müssten daher für unterschiedliche paradigmatische Zugänge sensibilisiert werden (etwa bei der Reflexion von Schulpraxis), damit sie Passungsverhältnisse zwischen ihrem individuellen Bildungsprozess und der paradigmatischen Verortung (Ich-WeltVerhältnis) herstellen können. Am Beispiel des Themenfeldes Inklusion wird gezeigt, warum eine mehrperspektivische Erschließung divergierender Positionen und Theorien bzw. Funktionen (z. B. Widerspruch von Inklusion und Selektion) erforderlich scheint. Auch die Lehrenden (Dozierenden) sind eine heterogene Gruppe; insbesondere seien sie in eine Methode oder ein Fach häufig so sehr einsozialisiert, „dass kein Sensus (mehr) für die Vielfalt der Paradigmen existiert" (S. 250). Dann aber existiere die Gefahr, dass Dozierende nur das eigene Paradigma lehren, ohne (partiell) Bezüge zu anderen Elementen der Lehrerbildung herzustellen. Schließlich erfordere bereits der Gegenstand notwendigerweise eine Multiparadigmatik. Spätestens wenn Lehrpersonen selektiv und willkürlich konkurrierende Begründungsmuster für ihre Handlungsentscheidungen unter Rekurs auf wissenschaftliche Theorie und Empirie heranziehen, erscheine eine informierte Sensibilität, ein „Wissen um die wechselseitige Beschränktheit, aber auch die wechselseitigen Möglichkeiten unterschiedlicher Forschungszugänge für die praktische Umsetzung" (S. 252), erforderlich.

\section{Meta-reflexive Lehrerbildung}

Den Ansatz der Meta-Reflexivität und seine auf die Lehrerbildung bezogenen Implikationen haben Cramer et al. (2019) entfaltet. Wie lassen sich die spezifischen Eigenlogiken bestimmter Paradigmen in der Lehrerbildung verstehen, in ihrem Verhältnis zueinander bestimmen und als kohärent für die institutionelle und individuelle Professionalisierung nutzen (Cramer \& Drahmann, 2019)? Im Modus der Meta-Reflexivität muss Professionalisierung auf verschiedenen Paradigmen beruhen, und Professionalität ist das Vermögen, sie vor dem Hintergrund generell mehr- 
deutiger (sozial-)wissenschaftlicher Erkenntnisse (Radtke, 1988, S. 106) zu reflektieren. Pädagogische Reflexion (wissenschaftliche Analyse pädagogischer Praxis) ist ein Ausgangspunkt, um Orientierungswissen für Handeln unter Ungewissheit zu generieren (Luhmann \& Schorr, 1982). Lehrerbildung scheint nur dann der Ungewissheit konsequent Rechnung zu tragen, wenn die Grundlagen der Reflexion im Reflexionsprozess mit bedacht werden. Dies erfordert das Heraustreten aus den mehrperspektivischen Beobachtungen, um im Sinne einer Beobachtung zweiter Ordnung die Mehrperspektivität selbst zu thematisieren.

Im Sinne eines solchen meta-kognitiven Vermögens kann Meta-Reflexivität verstanden werden als

„Kenntnis unterschiedlicher, auf den Lehrerberuf bezogener theoretischer Zugänge und empirischer Befunde, die Fähigkeit, diese mit Blick auf ihre jeweiligen Grundlagen und Geltungsansprüche verorten, in ein Verhältnis setzen und sich kritisch mit ihnen auseinandersetzen zu können sowie konsistente, exemplarisch-typisierende Deutungen des komplexen Handlungsfeldes Schule vornehmen zu können“ (Cramer et al., 2019, S. 410).

Unter Rekurs auf diese Deutungen können in der Schule letztlich situationsadäquate Handlungsoptionen entwickelt werden. Meta-reflexive (sekundäre) Lehrerbildung bezieht das primär gewonnene reflexive Fallverstehen und Professionswissen, die erworbenen Kompetenzen sowie die Sensibilität für (berufs-)biografisch relevante Ereignisse wechselseitig aufeinander. Dazu macht sie die den Ansätzen (und weiteren Theorien) immanenten Grundlagen und Argumente transparent.

Neun heuristische Prinzipien meta-reflexiver Lehrerbildung werden bislang angenommen (Cramer et al., 2019, S. 415): Konsequente Mehrperspektivität auf eine Fragestellung begünstigt das Entwickeln von Meta-Reflexivität. Auf ihrer Grundlage kann das Prinzip Distanzierung als Erkennen des Mehrwerts distanzierter (zeitlich entlasteter) Betrachtungen thematisiert werden. Die Kontextgebundenheit wissenschaftlichen Erkennens ist offenzulegen. Eine Thematisierung der Alternativität bzw. Vorläufigkeit allen Erkennens kann dafür sensibilisieren, dass das Handlungsfeld durch nur eine Perspektive nie hinreichend erklärt werden kann. Die Unabhängigkeit der Perspektiven ergibt sich aus unterschiedlichen Setzungen von Begriffen, Theoremen usw., weshalb sie in einem nicht-hierarchischen Verhältnis zueinander stehen. Transparenz in der Lehre ist erforderlich, indem eine Metakommunikation mit Studierenden über die Art und Weise erfolgt, wie Gegenstände meta-reflexiv betrachtet werden (können). Bezüglich der herangezogenen Quellen und der Relevanz der ausgewählten Theorien besteht eine Begründungspflicht Dozierender. Die Komplexität des Behandelten muss einer Dynamik unterliegen, um eine Überforderung Studierender zu vermeiden. 


\section{Gemeinsamkeiten und Differenzen}

Das Programm einer „multiparadigmatischen Lehrerbildung“ sowie der Ansatz der „meta-reflexiven Lehrerbildung“ weisen deutliche Gemeinsamkeiten auf, die sich insbesondere sowohl auf die Situationsanalyse der Lehrerbildung beziehen als auch auf die Absicht, Lehramtsstudierende im Umgang mit Mehrperspektivität in ihrer Professionalisierung zu unterstützen. Dies wird nun anhand ausgewählter Textzitate illustriert.

Die „Konfrontation Studierender mit einer Pluralität von (Erkenntnis-)Theorien, Wissensformen, Paradigmen und (Teil-)Disziplinen [ist] ein Spezifikum der Lehrerbildung“ (Cramer et al., 2019, S. 401). Inwieweit diese nicht zu Widersprüchen und Brüchen, sondern zu einer Inspiration im Professionalisierungsprozess führen, „hängt vom Grad der Verständigung oder zumindest wechselseitigen Wahrnehmung ab“ (Heinrich et al., 2019, S. 245). Doch Studierende „sind mit der Aufgabe bislang weitgehend auf sich alleine gestellt, diese unterschiedlichen Zugänge in ein stimmiges Gesamtbild zu überführen“ (Cramer et al., 2019, S. 401). Es ist „curricular fragwürdig, die Studierenden mit dieser Unübersichtlichkeit allein zu lassen. Erwartbar ist dann zumindest, dass sie diese unterschiedlichen Zugänge zu Wissenschaft nur schwerlich miteinander vermitteln können“ (Heinrich et al., 2019, S. 245). „Im Nachvollzug verschiedener Wege der Reflexion besteht ein Mehrwert“ (Cramer et al., 2019, S. 409). „Allerdings würde eine unmoderierte Ko-Existenz der Ansätze in separierten Modulen verhindern, dass die eigentlichen Dimensionen der Widersprüche sachgerecht gegenübergestellt und bearbeitet werden“ (Heinrich et al., 2019, S. 250). „Wird dieses Spannungsfeld ignoriert, so besteht die Gefahr der Nivellierung bzw. einseitigen Aufhebung der Komplexität solcher Spannungsverhältnisse“ (Cramer et al., 2019, S. 403), und es wird ignoriert, dass „jedem Paradigma in seinen Grenzen eine spezifische Funktion zukommt. [...] Notwendig ist damit ein wissenschaftstheoretischer Meta-Blick, der die unterschiedlichen Paradigmen in ihrer jeweiligen Eigenlogik zunächst begreifen und dann auch wertschätzen kann" (Heinrich et al., 2019, S. 252). „Meta-Reflexivität setzt damit Mehrperspektivität als notwendig voraus, es muss aber das Nachdenken über den Modus der mehrperspektivischen Betrachtung unter Ungewissheit hinzutreten“ (Cramer et al., 2019, S. 409).

Weiterhin zeigen sich Differenzen, die sich aufgrund der noch zu erfolgenden theoretischen Entfaltung der Multiparadigmatik allenfalls vorläufig skizzieren lassen:

(1) Epistemologische Begründung: Die Multiparadigmatik wird bislang zuvorderst aus den praktischen Erfordernissen der Lehrerbildung heraus sowie unter Rekurs auf das Grundmodell des didaktischen Dreiecks begründet. Meta-Reflexivität wird auf Basis professionstheoretischer, wissenschaftstheoretischer sowie ideengeschichtlicher Erwägungen fundiert. Dabei ist Meta-Reflexivität im Vergleich zur 
Selbstzuschreibung der Multiparadigmatik nicht „alternativlos“ (Heinrich et al., 2019, S. 243), sondern stellt ihrerseits einen möglichen Modus von Lehrerbildung dar.

(2) Umgang mit Widersprüchen: Multiparadigmatik wirft die Frage „Auswege aus dem Paradigmenstreit?“ (Heinrich et al., 2019, S. 243) auf, während Meta-Reflexivität im spannungsreichen Nebeneinander verschiedener Perspektiven ein konstitutives Moment der Lehrerbildung und eine besondere Chance sieht, Fragen nach Beruf, Schule und Unterricht in ihrer Relation zu erschließen. Zwar ist Paradigmenstreit auch aus meta-reflexiver Sicht unangemessen, doch sind die aus verschiedenen Paradigmen heraus erwachsenden Widersprüche eine Voraussetzung für perspektivenerweiternde Blickwendungen. Es ist gerade dieses „stabil instabile Verhältnis“ (Meseth \& Proske, 2018, S. 38) von z. B. Disziplin und Lehrerbildung, das ein Professionalisierungsmoment in sich birgt. Alle Auflösungsbemühungen von paradigmatischen Widersprüchen - sei es durch den Versuch der Durchsetzung einer monoparadigmatischen Sicht oder durch die pauschale Forderung nach Pluralismus - sind kritisch zu sehen.

(3) Verortung der Paradigmen: Zwar sieht auch die Multiparadigmatik im letzten Schritt der „multiparadigmatischen Reflexion die Ordnung der Erklärungsansätze“ (Heinrich et al., 2019, S. 252) vor; die Ordnungskriterien (z.B. Mikro-, Meso-, Makroebene des Bildungssystems, Erklärungsfokus: Profession oder Unterricht) werden aber von außerhalb an die Ansätze herangetragen. Meta-Reflexivität fokussiert noch stärker auf das Verstehen eines Paradigmas aus der Innensicht heraus und setzt dieses Durchdringen einer theoretischen und/oder empirischen Perspektive für die Fähigkeit voraus, sich dann kritisch und vergleichend-beobachtend dazu zu verhalten.

(4) Implikationen für die Lehrerbildung: Welche Implikationen Multiparadigmatik für Lehrerbildung hat, wird an konkreten Beispielen aufgezeigt, die im Kern allesamt auf die Relevanz einer „Mehrperspektivität“ verweisen. Für Überlegungen zur Meta-Reflexivität ist diese konsequente Mehrperspektivität der behandelten Gegenstände lediglich Ausgangspunkt didaktischer Überlegungen zur Lehrerbildung. Es treten die oben genannten (vgl. Kap. 3) Prinzipien meta-reflexiver Lehrerbildung (Distanzierung, Kontextgebundenheit, Alternativität usw.) hinzu.

(5) Limitationen: Die für die Meta-Reflexivität angenommenen Limitationen (Cramer et al., 2019, S. 418), etwa die drohende Überforderung oder - entgegen der eigentlichen Absicht - Desorientierung Studierender durch Konfrontationen mit komplexen (wissenschaftstheoretischen) Überlegungen und Implikationen, dürften auch für die Multiparadigmatik gelten und werden dort bislang noch nicht diskutiert. 


\section{Perspektiven}

Mit dem Erscheinen der Beiträge zur Multiparadigmatik und Meta-Reflexivität wird letztlich ein sowohl in der Universitätsgeschichte als auch in der Diskussion um Lehrerbildung virulenter Diskus um das Verhältnis unterschiedlicher Paradigmen aufgenommen. Die Notwendigkeit einer wieder verstärkten Auseinandersetzung mit der Herausforderung, dass es für komplexe Fragen nach Schule und Unterricht in der Lehrerbildung keine einfachen Antworten geben kann, erscheint allerdings für Studierende und Dozierende von neuer Relevanz: Gerade durch die Ausfinanzierung umfangreicher Programme wie der „Qualitätsoffensive Lehrerbildung“ in Deutschland oder der „PädagogInnenbildung NEU“ in Österreich werden Maßnahmen ergriffen, die schnellen Erfolg bei der Optimierung der Lehrerbildung versprechen (müssen), die aber auch zu einer Deprofessionalisierung beitragen können, wenn sie informell-individuelle Kohärenz in der Lehrerbildung seitens der Studierenden (und Dozierenden) durch Aufsummieren von unverbundenen Einzelmaßnahmen eher verhindern als unterstützen. Lehrerbildung an der Universität kann daher alleine schon aus Kohärenzgesichtspunkten nicht auf wissenschaftlichen Diskurs verzichten, der sich auch durch Streit in der Sache auszeichnet, ohne das Eigene als alleinig „relevant", „wahr“ oder gar „wissenschaftlich“ zu klassifizieren.

Dieses Anliegen betonen die Ansätze Multiparadigmatik und Meta-Reflexivität eindrücklich und lehnen eine unterkomplexe Lehrerbildung ab. Eine Lehrerbildung, die sich durch monoparadigmatische Tendenzen oder einseitige Leitansprüche charakterisiert, verkennt das Potenzial von Blickwendungen, die zuvorderst in einer multiparadigmatischen bzw. interdisziplinären Gemengelage $\mathrm{zu}$ Tage treten. Sie wäre indes mehr oder ausschließlich ein politisches, aber kein wissenschaftliches (universitäres) Unterfangen oder gar ein Bildungsprozess. Auch erscheint es kaum ausreichend, nur eine Mehrperspektivität zu pflegen, also das Nebeneinander verschiedener Perspektiven der Welterschließung zu praktizieren; es bedarf auch deren meta-reflexiver Durchdringung: Neben das Erkennen der Möglichkeit mehrperspektivischer Betrachtungen und die Fähigkeit, verschiedene Perspektiven einnehmen zu können, tritt die Notwendigkeit der Erklärung, worin sich diese Perspektiven unterscheiden. Der Gewinn einer meta-reflexiven Betrachtung besteht darin, Relationen zwischen den Perspektiven herzustellen und diese je kontext- und situationsspezifisch bei der Interpretation oder Reflexion schulischer Handlungssituationen abzuwägen. Dieses Ansinnen ist auch durch das Programm der „Multiparadigmatik“ beabsichtigt, ist aber dem Begriff nicht immanent, weil dieser letztlich lediglich auf Mehrperspektivität rekurriert. Der Begriff der „Meta-Reflexivität“ erlaubt durch die Verwendung des meta, explizit auszuweisen, dass neben der mehrperspektivischen Betrachtung auch die metakognitive Leistung eines Heraustretens aus den jeweiligen Perspektiven bedeutsam ist und sich mit dieser spezifischen Form von Reflexivität ein Aspekt von Professionalität im Lehrer*innenberuf verbindet. 


\section{Literatur und Internetquellen}

Böhme, J., Cramer, C., \& Bressler, C. (Hrsg.). (2018). Erziehungswissenschaft und Lehrerbildung im Widerstreit!? Verhältnisbestimmungen, Herausforderungen und Perspektiven. Bad Heilbrunn: Klinkhardt.

Cramer, C. (2018). Zum Verhältnis von Erziehungswissenschaft und Lehrerbildung. Implikationen für die Professionalität im Lehrerinnen- und Lehrerberuf. In J. Böhme, C. Cramer \& C. Bressler (Hrsg.), Erziehungswissenschaft und Lehrerbildung im Widerstreit!? Verhältnisbestimmungen, Herausforderungen und Perspektiven (S. 103-118). Bad Heilbrunn: Klinkhardt.

Cramer, C., \& Drahmann, M. (2019). Professionalität als Meta-Reflexivität. In M. Syring \& S. Weiß (Hrsg.), Lehrer(in) sein - Lehrer(in) werden - die Profession professionalisieren (S. 17-33). Bad Heilbrunn: Klinkhardt.

Cramer, C., Harant, M., Merk, S., Drahmann, M., \& Emmerich, M. (2019). Meta-Reflexivität und Professionalität im Lehrerinnen- und Lehrerberuf. Zeitschrift für Pädagogik, 65 (3), 401-423.

Glowinski, I., Borowski, A., Gillen, J., Schanze, S., \& von Meien, J. (2018). Kohärenz in der universitären Lehrerbildung. Potsdam: Universitätsverlag Potsdam.

Heinrich, M., Wolfswinkler, G., van Ackeren, I., Bremm, N., \& Streblow, L. (2019). Multiparadigmatische Lehrerbildung. Produktive Auswege aus dem Paradigmenstreit? DDS - Die Deutsche Schule, 111 (2), 244-259. https://doi.org/10.31244/dds.2019.02.10

KMK (Sekretariat der Ständigen Konferenz der Kultusminister der Länder in der Bundesrepublik Deutschland) (2004). Standards für die Lehrerbildung. Bildungswissenschaften. Beschluss der Kultusministerkonferenz vom 16.12.2004. Zeitschrift für Pädagogik, 51 (2), 280-290.

Luhmann, N., \& Schorr, K. E. (1982). Das Technologiedefizit der Erziehung und die Pädagogik. In N. Luhmann \& K. E. Schorr (Hrsg.), Zwischen Technologie und Selbstreferenz. Fragen an die Pädagogik (S. 11-40). Frankfurt a. M.: Suhrkamp.

Meseth, W., \& Proske, M. (2018). Das Wissen der Lehrerbildung zwischen Wissenschaftsund Praxisorientierung. In J. Böhme, C. Cramer \& C. Bressler (Hrsg.), Erziehungswissenschaft und Lehrerbildung im Widerstreit!? Verhältnisbestimmungen, Herausforderungen und Perspektiven (S. 19-43). Bad Heilbrunn: Klinkhardt.

Radtke, F.-O. (1988). Professionelles Halbwissen. Tabus über die Lehrerbildung. Neue Sammlung, 28 (1), 93-108.

Terhart, E. (2000). Perspektiven der Lehrerbildung in Deutschland. Abschlussbericht der von der Kultusministerkonferenz eingesetzten Kommission. Weinheim: Beltz.

Colin Cramer, Prof. Dr., geb. 1979, Lehrstuhlinhaber für Professionsforschung unter besonderer Berücksichtigung der Fachdidaktiken an der Eberhard Karls Universität Tübingen.

Korrespondenzadresse: Tübingen School of Education, Wilhelmstraße 31, 72074 Tübingen

E-Mail: colin.cramer@uni-tuebingen.de 